\title{
Gestação de Termo após Ablação Endometrial
}

\author{
Full-term Pregnancy after Endometrial Ablation
}

Rievane S. Damião, Frederico F. Rodrigues, Salete Yatabe José Francisco D. Ramos, Reginaldo G. C. Lopes, Umberto G. Lippi

\section{RESUMO}

\begin{abstract}
A ablação endometrial por via histeroscópica é uma técnica utilizada em casos de sangramento uterino anormal com resposta desfavorável ao tratamento clinico. Mesmo com destruição ou ressecção das camadas funcional e basal do endométrio é possivel ocorrer uma gravidez posterior à intervenção. No entanto essas gestações freqüentemente terminam em abortamento ou se apresentam com complicações. Relata-se o caso de uma paciente que desenvolveu gravidez a termo sem intercorrências após ter realizado ablação endometrial por sangramento uterino anormal resistente ao tratamento clinico.
\end{abstract}

PALAVRAS-CHAVE: Sangramento uterino disfuncional. Ablação do endométrio. Complicações da gravidez.

\section{Introdução}

A ablação endometrial por via histeroscópica foi introduzida no início da década de 80. Está indicada nas pacientes com diagnóstico de sangramento uterino anormal (SUA), geralmente em útero sem anomalia anatômica, resistente ao tratamento clínico ou diante de contra-indicação para este ${ }^{5}$. Por meio dessa intervenção resseca-se ou destroem-se as camadas funcional e basal do endométrio.

Dentre as várias técnicas utilizadas uma das mais comuns é a de Hamou ${ }^{1}$, que mantém a camada endometrial cerca de $1 \mathrm{~cm}$ acima do orificio interno. Com esta técnica geralmente não há o acolamento total das paredes uterinas e torna-se possivel a realização de uma histeroscopia diagnóstica ulterior, se necessário.

Embora a gravidez após a ablação endometrial seja um evento de baixa freqüência, pode ocorrer em 0,4 a $1 \%$ dos casos de ablação ${ }^{2,3,5}$ No entanto, essas gestações tendem a apresentar complicações tais como inserção baixa de placenta,

Serviço de Ginecologia e Obstetrícia do Hospital do Servidor Público Estadual "Francisco Morato de Oliveira " - IAMSPE SP (HSPE-FMO)

Correspondência:

Umberto Gazi Lippi

Rua Borges Lagoa 564, cj 32 - Vila Clementino

04038-000 - São Paulo - SP acretismo placentário, trabalho de parto prematuro e muitas terminam em abortamento ${ }^{6}$.

Relata-se a seguir um caso de ablação endometrial seguido de gravidez a termo, isenta de complicações ou intercorrências.

\section{Relato do caso}

CRIP, 40 anos, natural e procedente de São Paulo, foi encaminhada ao Hospital com quadro de SUA (ciclos menstruais hipermenorrágicos) evoluindo há 12 meses. Apresentava antecedente obstétrico de cinco gestações, com dois partos normais e três abortamentos espontâneos. Os exames físico e ultra-sonográfico não revelaram anormalidades dos órgãos genitais. À histeroscopia foi observado aumento da cavidade uterina, com algumas traves fibróticas entre as paredes anterior e posterior. Os óstios tubários não foram visualizados e o endométrio era do tipo secretor, confirmado, posteriormente, por biópsia orientada. O diagnóstico histeroscópico foi de sinéquias uterinas.

A paciente recebeu $10 \mathrm{mg}$ de medroxiprogesterona ao dia, na segunda fase do ciclo, durante 3 meses, sem melhora do quadro clínico. Foi indicada ablação endometrial, após a administração de análogos do GnRH por 12 semanas. A cirurgia foi realizada com a técnica de redução endometrial, 
segundo preconizado por Hamou ${ }^{1}$, preservando-se o endométrio da região $1 \mathrm{~cm}$ acima do orifício interno. A cirurgia transcorreu dentro da normalidade com alta hospitalar em 12 horas. $\mathrm{O}$ exame histopatológico da ablação endometrial revelou hiperplasia simples do endométrio, sem atipia. Foi proposta a esterilização, mas a paciente não aceitou. Mostrou-se, então, a importância de adotar algum método anticoncepcional, esclarecendo-a sobre o eventual risco de gestação pós-ablação.

A paciente evoluiu com melhora do quadro clínico, apresentando ciclos hipomenorrágicos. Após 4 meses, a paciente retornou queixando-se de sangramento inter-menstrual, tipo "spotting", e foi submetida a nova histeroscopia. Em virtude da queixa excessiva de dor na introdução do histeroscópio, associada à realização de um falso trajeto, optou-se por adiar o exame. Decorridos mais três meses a paciente procurou novamente o serviço com suspeita de gravidez, confirmada por ultra-sonografia. Encaminhada para o pré-natal de gestação de alto risco houve evolução até o termo sem complicações e/ou intercorrências obstétricas. Foi submetida a cesárea originando um recémnascido vivo, com peso de $4.210 \mathrm{~g}$ e escores de Apgar de 9 e 10, respectivamente ao primeiro e ao quinto minutos. A dequitação foi normal. Não foram observadas intercorrências neonatais.

\section{Comentários e conclusões}

A técnica de redução do endométrio adotada no Setor de Endoscopia Ginecológica do HSPE-FMO é a descrita por Hamou ${ }^{1}$. Consiste na ressecção do endométrio funcional e basal, deixando um remanescente de $1 \mathrm{~cm}$, a partir do orificio interno do colo uterino.

Uma das preocupações que deve ser considerada pelos ginecologistas é a possibilidade de gestação após a ablação do endométrio. Quando esta ocorre, há, em geral, maior risco pré-natal explicado pelo fato de a ablação endometrial causar sinéquias uterinas, que dificultam a implantação adequada do ovo. Isto pode resultar em complicações, tais como: abortamento, descolamento prematuro de placenta, placenta prévia, placenta increta ou percreta, rotura uterina, trabalho de parto prematuro e restrição do crescimento intra-uterino (RCIU) ${ }^{4}$.

Mongelli e Evans ${ }^{6}$, analisando 2585 ablações de endométrio, encontraram 26 gestações, sendo que 19 pacientes evoluíram para abortamento no primeiro trimestre e as sete restantes para parto a termo, com incidência elevada de RCIU. Três dessas gestantes apresentaram inserção anormal de placenta, e houve a necessidade de se realizar histerectomia puerperal em um dos casos.

No Setor de Endoscopia Ginecológica do HSPE-FMO, entre 1991 e 1999, foram realizadas 78 ablações de endométrio, com apenas 1 caso de gestação pós-ablação (1,2\%).

A incidência de gestação após ablação varia na literatura mundial entre 0,4 e $1 \%$ de acordo com a técnica preconizada ${ }^{2,3,5}$. Magos et al. ${ }^{3}$ relataram uma incidência de $0,4 \%$, com maior prevalência entre as pacientes que realizaram a ressecção parcial, à semelhança do que se faz neste Serviço.

A preocupação em manter a cavidade uterina permeável quando adotada a técnica de redução do endométrio justifica-se em virtude da possibilidade de se necessitarem novas investigações endometriais futuras. Por outro lado, estas pacientes devem ser incisivamente alertadas quanto à necessidade de uso de um método anticoncepcional contínuo e eficaz, talvez até uma laqueadura por via laparoscópica, pois uma gestação nem sempre apresentará evolução favorável.

\section{Summary}

Endometrial ablation is a useful technique in patientes with abnormal uterine bleeding without response to clinical measures. Pregnancy is possible even after the destruction or resection of the endometrium. The case reported is a normal term pregnancy after endometrial ablation because ot menorrhagia without successful prior clinical treatment.

KEY WORDS: Endometrial ablation. Pregnancy complications

\section{Referências bibliográficas}

1. Hamou J. Hysteroscopy and microcolpohysteroscopy. Text and Atlas. New York: Appleton \& Lange; 1991.

2. Hill DJ, Maher PJ. Pregnancy following endometrial ablation. Gynecol Endosc 1992, 1: 47-49.

3. Magos AL, Baumann R, Lockwood GM, Turnbull AC. Experience with the first 250 endometrial resections for menorrhagia. Lancet 1991; 337: 1074-8.

4. McLucas B. Pregnancy after endometrial ablation: A case report. J Reprod Med 1995; 40:237-9.

5. Mencaglia L, Tantini C. Ablação endometrial. In: J Ueno, Nc Santos, W Pinheiro, JA Pinotti, editores. Cirurugia vídeo endoscopia em ginecologia. São Paulo: Roca, 1997. p.353.

6. Mongelli JM, Evans AJ. Pregnancy after transcervical endometrial resection. Lancet 1991; 338: 578-9. 